\title{
Study on Underwater Laser Scribing Technique
}

\author{
Yuhong Long ${ }^{1, \mathrm{a}}$, Youqun Tong ${ }^{1, \mathrm{~b}}$, Jiading $\mathrm{Bao}^{1, \mathrm{c}}$, and Tanggao Feng ${ }^{1, \mathrm{~d}}$ \\ ${ }^{1}$ School of Guilin University Of Electronic Technology, Guilin 541004, China; \\ alongyuhong@guet.edu.cn, btongyouquan@qq.com, c38792839@qq.com, d2429052125@qq.com
}

Keywords: underwater laser, melt splashing, smooth particle dynamics.

Abstract. In order to make further explorations to the mechanism of underwater laser scribing, comparison of laser scribing in air and underwater under different power is conducted in this paper, at the same time simulation to melt splashing based on smooth particle dynamics is carried out. The results show that the underwater laser processing can effectively reduce the heat affected zone, to avoid forming a metamorphic layer. Under large power, efficiency of scribing is effectively improved due to the plasma shock wave produced underwater and cavitation phenomenon and chip removal effect of the water wave.

\section{Introduction}

As silicon is a kind of hard-brittle material, contacting process is easy to cause crack and edge damage. Due to being non-contacting processing, the laser has incomparable advantages comparing with traditional machining [1]. In recent years, with the continuous development of laser devices and processing technology, its application scale rapidly expanded. Laser scribing has been used for many years. Its application showed well in low price products, but its quality had been unable to be recognized in the processing of integrated circuits due to its excessive heat affected zone [2]. In order to reduce heat affected zone, this paper proposed laser and solution compound processing technology, among which the most common are underwater laser processing, water guided laser processing and water jet laser processing. Water guided laser processing has the most superior performance, but the technology is complex and expensive, and the patent is monopolized by the foreign countries [3]. Underwater laser processing technology is simpler, having obvious advantages compared to that in air. In this paper, experiment and the melt splashing simulation of the underwater laser processing is conducted.

\section{Experiment Research}

\section{Experimental Conditions}

The laser used in this experiment is YMS - 20F fiber laser scribing machine produced in Zhuhai Yue Mao Laser Equipment Engineering Co., Ltd, with a power rating of 20W, basic mode as laser output mode, optical quality $\mathrm{M}^{2}<1.3$, and electro-optic conversion efficiency of 35\%. Experiments mainly studied the appearance and quality on the surface of silicon wafers after scribing in the air and water. The experimental schematic diagram is shown in Fig.1.

The silicon wafer used in experiment has smooth surface and uniform thickness of $725 \mathrm{um}$. Water layer thickness is set for $2.5 \mathrm{~mm}$ to achieve good cooling and chip removal effect, and reduce the attenuation of the laser. This experiment will be divided into four groups, and the experimental parameters are as follows. In the first group, the laser output power is $20 \mathrm{~W}$, scribing in the air; in the second group, the laser output power is $12 \mathrm{~W}$, scribing in the air; in the third group the laser output power is $20 \mathrm{~W}$, scribing underwater; in the fourth group, the laser output power is $12 \mathrm{~W}$, scribing underwater. Based on the principle of control variables, repetition frequency is $100 \mathrm{kHz}$, pulse width is 25 to 50ns, cutting speed is $10 \mathrm{~mm} / \mathrm{s}$, and feeding is twice. When laser cuts in the air, sparks can be seen. When laser cuts underwater, a lot of bubbles come up in the cutting area. 


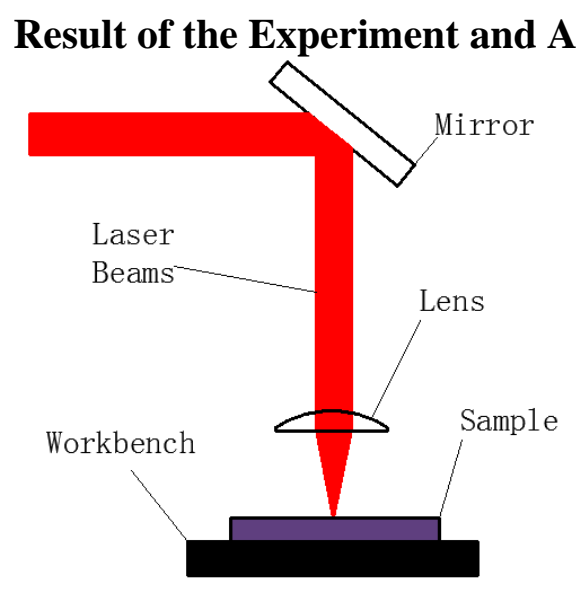

a

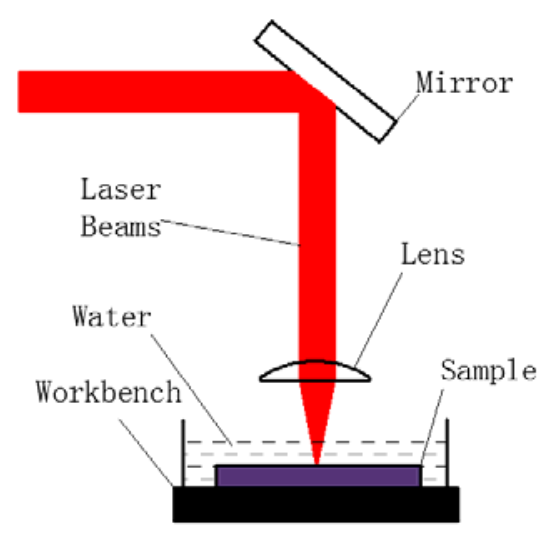

b

Fig.1 Schematic diagram of laser scribing (a) in air, (b) underwater.

Use Quanta200 scanning electron microscope to observe and analyze the surface microstructure of the scribing area. The results is shown in Fig.2.

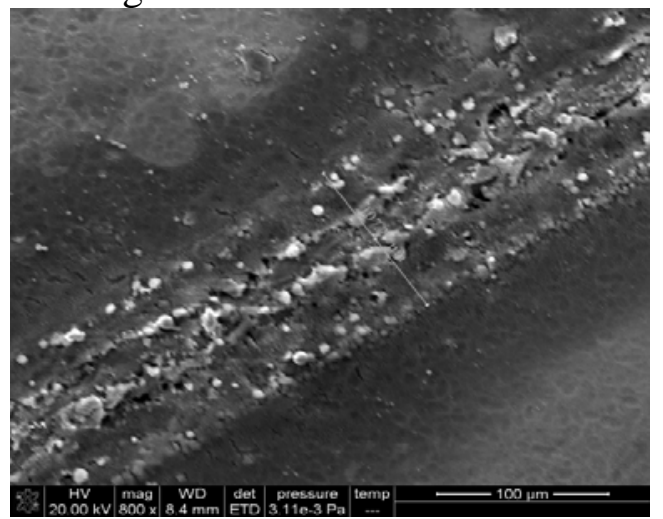

a

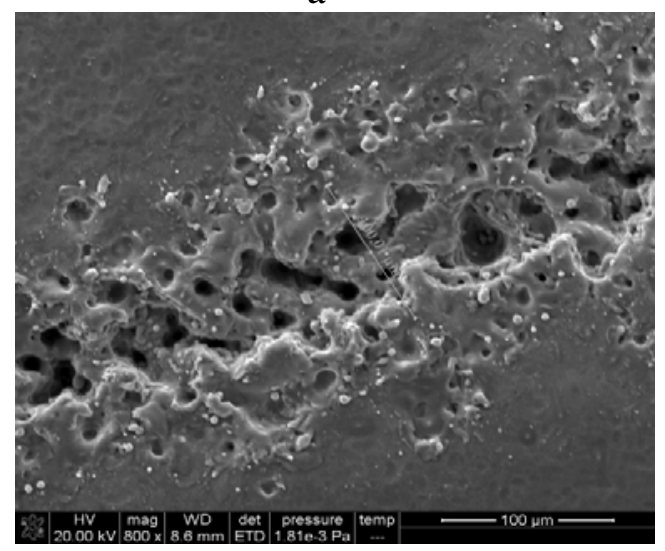

C

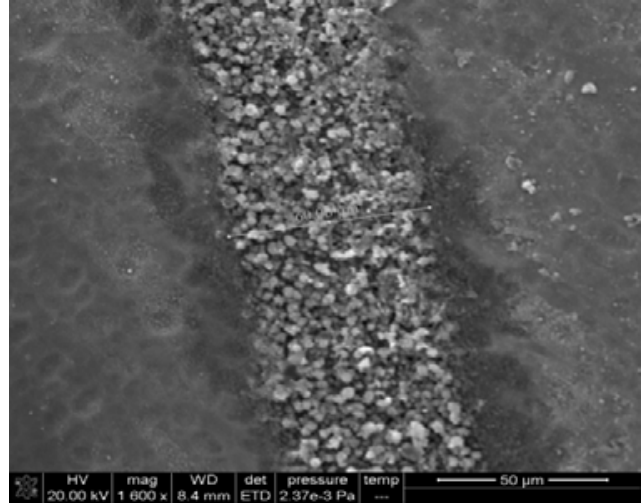

b

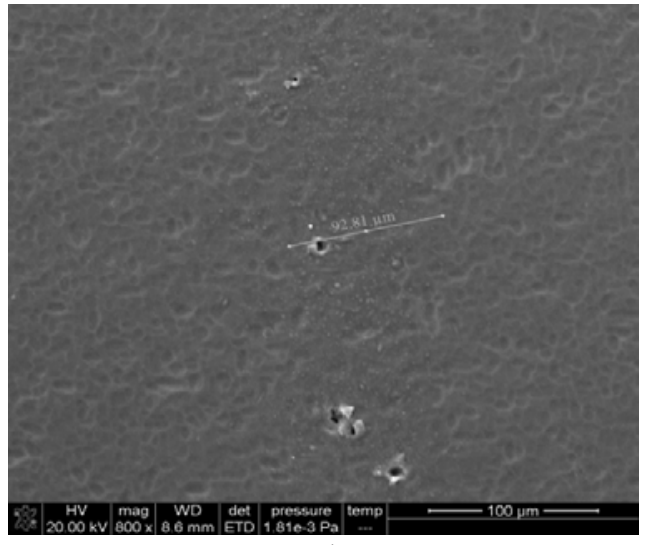

d

Fig.2 Scanning electron microscope picture of scribing samples (a) in air at a power of 20W, (b) in air at power of $12 \mathrm{~W}$, (c) underwater at power of $12 \mathrm{~W}$, (d) underwater at power of $12 \mathrm{~W}$.

Comparing Fig.2a and Fig.2c, it can be found that in the laser power of 20W, underwater laser cutting depth is deeper with obvious material removal effect. A lot of holes in the cutting area are formed. This is because that when the high energy laser acts on the wafer surface in water, optical breakdown happens at the laser focus area, resulting high temperature and high pressure plasma, which absorbs the following laser energy, forming the outward plasma shock wave. At the same time, when laser breaks down some material, cavitation phenomenon occurs. When there are solid walls around bubbles, the bubbles break, forming high-speed jet pointing to target. The impact generated by high-speed jet can reach to grade of $\mathrm{MPa}$ [4]. Elastic deformation on silicon surface will be formed under the action of plasma shock wave and jet velocity. Secondly due to the interaction between 
shock wave and the molten material splashing, water fluctuation occurs, taking away a large number of the melt. In the process water plays a role in chip removal, taking away the melt, thus forming pits and holes. Therefore, it can be seen that when the laser power is large, the water layer can promote the removal of material to improve the machining efficiency.

Comparing Fig.2b and Fig.2d, it can be found that, in the laser power of 12W, underwater laser cutting trace is not obvious, because the pure water absorbs part of the laser thus causes energy attenuation, which can be calculated by the Lambert-Beer's law. The fluctuation of water during processing also can produce refraction of laser causing energy attenuation.

Comparing Fig.2a, Fig.2b, Fig.2c, Fig.2d, it can be found that processing area in the air showed black with heavy residue resolidification under electron microscopy. While no obvious black and residue resolidification appears in the processing in water. The black part is metamorphic layers caused by high temperature, and part of which is the oxide. Because the water had the effect of cooling and isolation of oxygen, the underwater laser scribing can effectively avoid the qualitative change caused by laser processing.

According the above, it can be seen that when the laser energy is high, water layer can improve the processing efficiency. When the laser energy is low, water layer mainly reduce the laser energy, preventing the processing. At the same time, water layer can effectively avoid the qualitative change and residue resolidification produced by laser machining, improving the processing quality.

\section{Melt splashing simulation}

In scribing, boiling in the melt layer of silicon wafer is quite fierce. The temperature rose sharply in a short period of time, which causes internal pressure of melting layer increase significantly. In order to release the pressure, a similar explosion of molten material splashing is formed. To further explore the interaction between melt splashing and water, this paper conduct a simulation of melt splashing based on smooth particle dynamics.

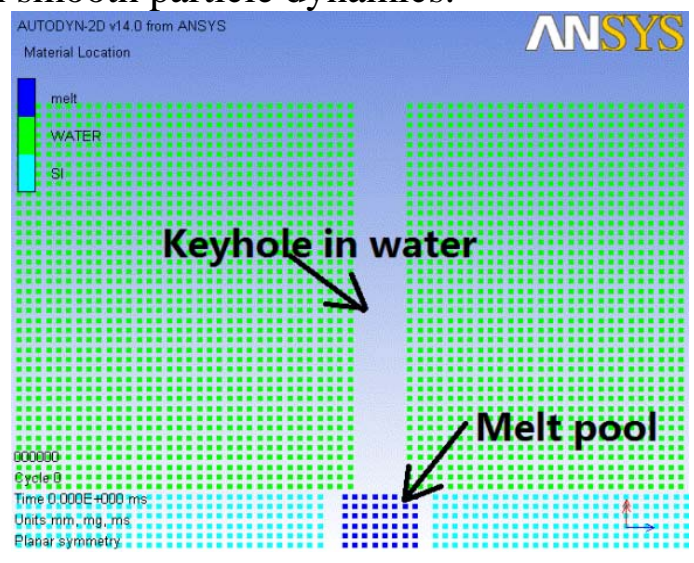

a

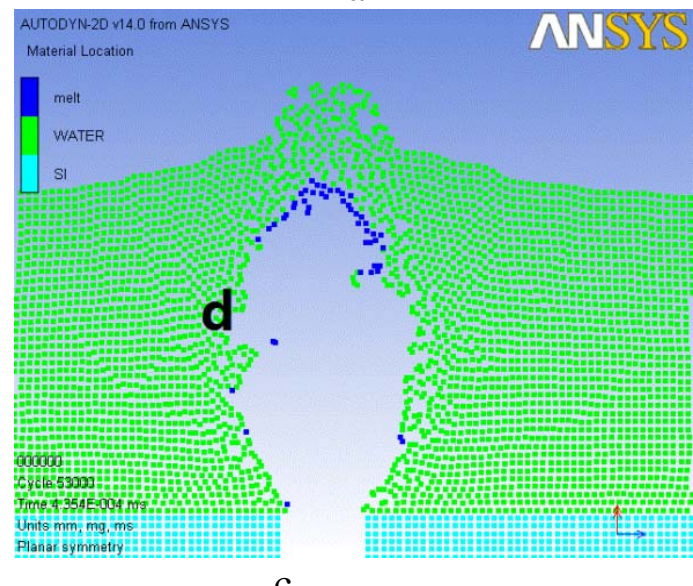

C

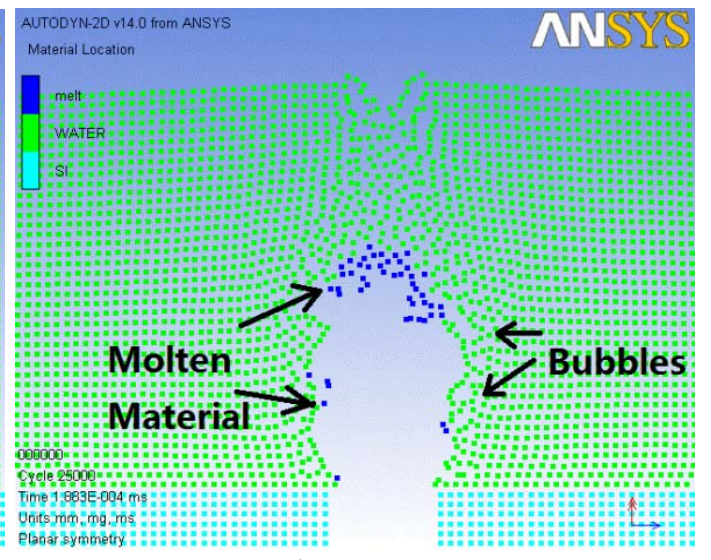

$\mathrm{b}$

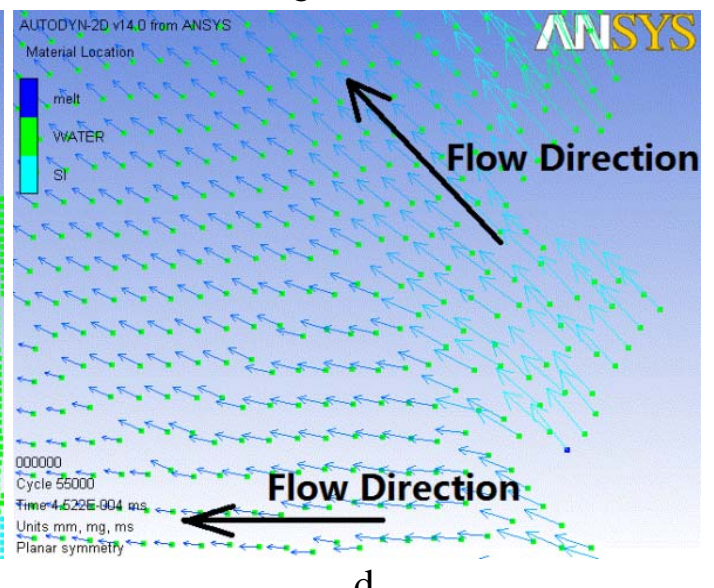

d

Fig.3 Time history of melt ejection during underwater laser scribing and the overview vector plot. 
Due to the complexity of the laser cutting and the limitation of the software, model was correspondingly simplified, without considering the effect of laser energy, assuming that the melt has been formed, and began to splash out under the internal pressure of the molten layer [5]. In Figure3, a, b, c show the melt splashing process. Fig.3b shows that when the melt splashes, most of the melt gather together, and a small part of melt disperse with water bubbles. Fig.3c shows that most of the melt spread out on both sides in the process of interaction with water, at the same time, ripples appear on the surface of the water due to melt splashing. Take magnification on the flow field at $d$ of Fig.3c, we can see laminar flow appear in the central layer, where laminar flow stimulated by melt splashing can take away the melt. Simulation is accorded with experiment, that is, the water can get rid of the melt, playing a role in chip removal. In the upper portion of the water, water inclines upward, thus to form ripples on the surface of water, which will reduce energy of underwater laser. SPH provides a straightforward simulation of the melt splashing process and the characteristics of the flow field, showing that the water layer benefits to the removal of the melt.

\section{Conclusions}

(1) Through the experimental analysis of the effect of laser scribing in air and underwater, the results show that underwater laser scribing can effectively avoid forming the metamorphic layer.

(2) Under large power water layer can improve the efficiency of laser scribing. Under low power, the water will absorb the laser energy, block processing.

(3) In addition, this paper conducted simulation to melt splashing based on smooth particl dynamics. The simulation results conform to the experimental results, that is, water layer can play a role in chip removal, which verified the feasibility of the simulation.

\section{Acknowledgements}

This work was financially supported funded by National Natural Science Foundation of China (61366009),the Innovation Project of GUET Graduate Education(GDYCSZ201444), the key project of Guangxi Natural Science Foundation (2015GXNSFDA139036), the director Fund of Guangxi Manufacturing Systems and Advanced Manufacturing Technology Laboratory (13-051-09-004Z), and Guangxi Experiment Center of Information Science, Guilin University of Electronic Technology (20130313).

\section{References}

[1]. Wei Yang, Xinhan Peng, Jun Zang, et al. Study of Laser cutting technology for silicon wafer under water(In Chinese). Chinese Journal of Lasers. Vol. 36 (2009) No. 11, p. 3064-3067.

[2]. Fumin Huang, Xiaozhu Xie, Xin Wei, et al. Newly developed technique for laser dicing wafer (In Chinese). Laser Tchnology. Vol.36,(2012)No. 3 p. 293-296.

[3]. V.Tangwarodomnukun, J.Wang, C.Z.Huang, et al. Heating and material removal process in hybrid laser-waterjet ablation of silicon substrates. International Journal of Machine Tool \& Manufacture. Vol. 79(2014), p. 1-16.

[4]. Lingrui Kong, Fei Zhang, Jun Duan, et al. Research of water-assisted laser etching of alumina ceramics(In Chinese). Laser Technology. Vol. 38 (2014) No. 3, p. 330-334.

[5]. Yinzhou Yan, LinLi, Kursad Sezer, et al. $\mathrm{CO}_{2}$ laser underwater machining of deep cavities in alumina. Journal of the European Ceramic Society. Vol.31(2011) ,p.2793-2807. 